\title{
Relapse of Yellow Nail Syndrome with Pulmonary Lymphedema
}

\author{
Kozo Morimoto ${ }^{1}$, Takeshi Saraya ${ }^{2}$, Atsuko Kurosaki ${ }^{3}$, Ryozo Yano ${ }^{1}$, Yuka Sasaki ${ }^{1}$, \\ Takeshi Osawa ${ }^{1}$, Shoji Kudoh ${ }^{1}$ and Hajime Goto ${ }^{1}$
}

\begin{abstract}
Yellow nail syndrome (YNS) is a rare disorder characterized by the triad of yellow, thickened nails, lymphedema, and respiratory manifestations such as pleural effusions, bronchiectasis, and recurrent lower respiratory tract infections. We report a case of YNS showing pulmonary interlobular septal thickening on thoracic computed tomography, implying the presence of lymphatic edema. The patient showed both the remission and relapse of yellow nail with different lung treatments over a long clinical course.
\end{abstract}

Key words: yellow nail syndrome, respiratory manifestations, interlobular septal thickening, lymphedema

(Intern Med 55: 169-172, 2016)

(DOI: 10.2169/internalmedicine.55.4849)

\section{Introduction}

Yellow nail syndrome (YNS) is a rare disorder that was first described by Samman and White in 1964 (1). It is characterized by the triad of thickened yellow nails, lymphedema in the extremities, and respiratory manifestations. However, no reports have described lymphedema in the lungs with the concomitant presence of accentuated interlobular septal thickening. We herein present a case of YNS showing pulmonary lymphedema with interlobular septal thickening.

\section{Case Report}

A previously healthy 65 -year-old man was referred to our hospital with progressive dyspnea, general fatigue, and a loss of appetite, which had developed over a period of several days before his presentation. He had noticed the slow yellowing of his nails from around 9 months prior to his presentation. He was an office worker and denied any use of illicit drugs or contact with ill individuals. Physical examinations revealed no abnormalities other than the yellowish discoloration and thickening of all of the fingernails (Fig. 1A), and fine crackles in the bilateral posterior lower lung fields. At first sight, his vital signs appeared normal, with the exception of a low-grade fever $\left(37.1^{\circ} \mathrm{C}\right)$, mild tachypnea ( 82 beats/min), and hypoxemia [percutaneous oxygen saturation $\left(\mathrm{SpO}_{2}\right) 85 \%$ with an ambient air]. The results of serum laboratory examinations were normal, with the exception of a moderate elevation of the serum Creactive protein level $(16.95 \mathrm{mg} / \mathrm{dL})$. The rheumatoid factor and antinuclear antibody results were both negative. Thoracic computed tomography (CT) showed non-segmental consolidation, ground-glass opacities, and traction bronchiectasis within the consolidation in the bilateral lower lung lobes (Fig. 2A). On suspicion of pneumonia, microbiological examinations were performed, but no pathogens were isolated from the sputum or blood. In addition, bronchoalveolar lavage (BAL) was performed on the day of admission, but the fluid cultures showed no pathogens. A BAL fluid analysis showed an elevated total cell count of $3.7 \times 10^{6}$ cells (neutrophils, 0\%; eosinophils, 0\%; lymphocytes, 21.8\%; macrophages, $78.2 \%$ ) and a decreased $\mathrm{CD} 4 / 8$ ratio of 1.6. A transbronchial lung biopsy (TBLB) performed for a pathological assessment on hospital day 14 revealed organizing changes, confirming the findings of the thoracic CT. Based on these findings, the patient was tentatively diagnosed with cryptogenic organizing pneumonia (COP). The patient was treated with intravenous methylprednisolone (1

${ }^{1}$ Respiratory Disease Center, Fukujuji Hospital, Japan Anti-Tuberculosis Association, Japan, ${ }^{2}$ Department of Respiratory Medicine, Kyorin University, Japan and ${ }^{3}$ Department of Clinical Radiology, Fukujuji Hospital, Japan Anti-Tuberculosis Association, Japan

Received for publication December 29, 2014; Accepted for publication April 26, 2015

Correspondence to Dr. Kozo Morimoto, morimotok@fukujuji.org 


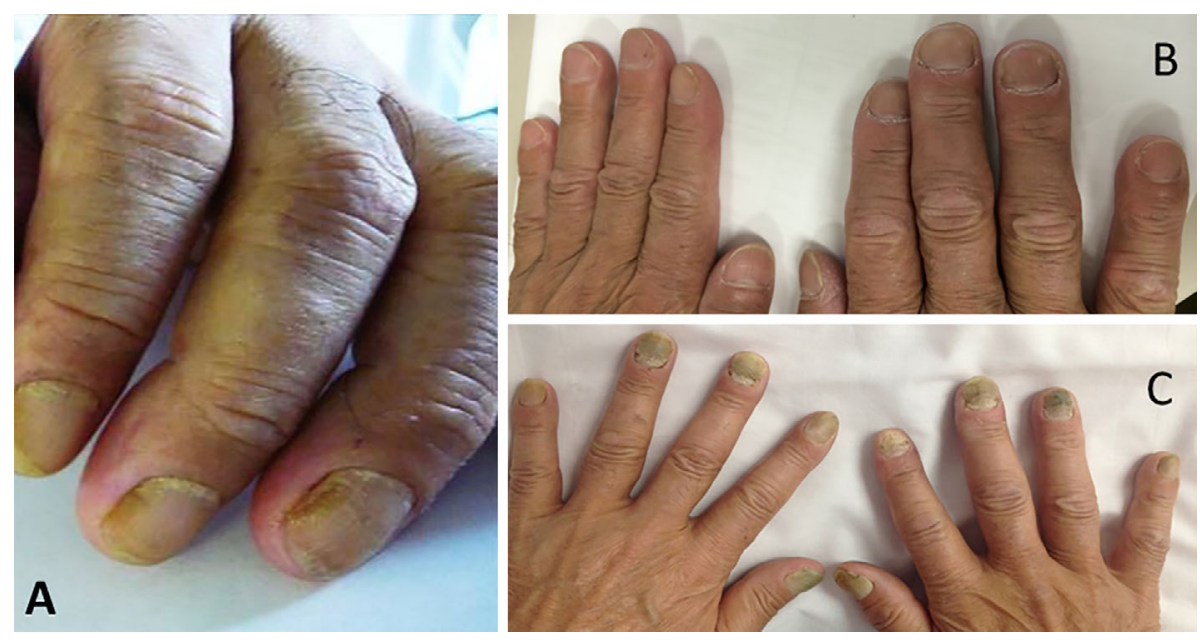

Figure 1. A yellowish discoloration of the nails is apparent on the left hand on the patient's first admission (A). This was completely resolved within 1 year after discharge (B). At 4 years after discharge, the nails on both of the patient's hands are thickened and show a more densely yellow discoloration (C).
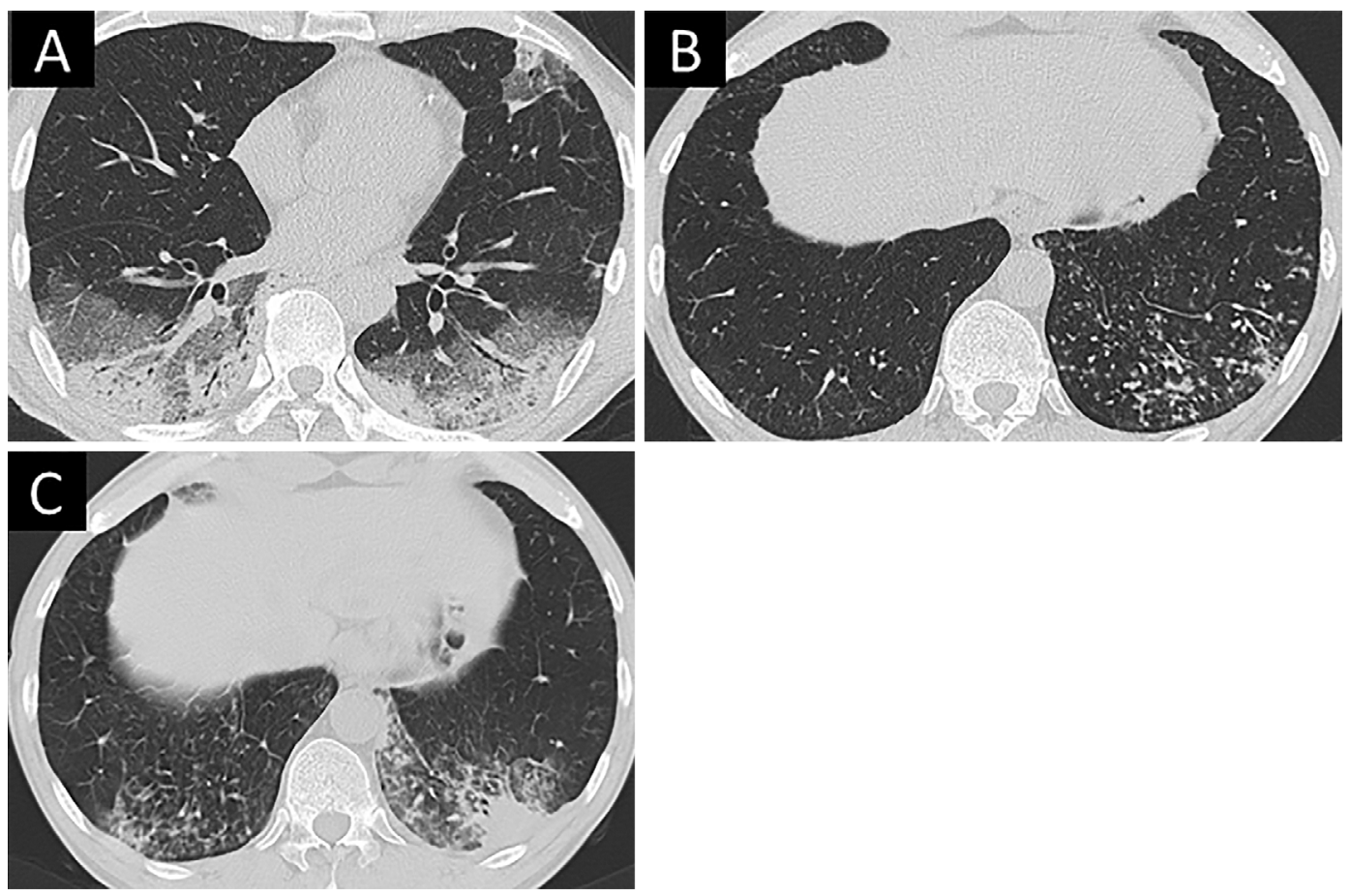

Figure 2. Chest computed tomography on the first admission shows non-segmental consolidation with ground-glass opacities in the bilateral lower lung lobes (A). Centrilobular nodules are noted in the left lower lobes in the outpatient clinic at 1 year after discharge $(B)$, and consolidation with ground-glass opacities is apparent 3 years after discharge $(C)$.

$\mathrm{g} /$ day for 3 consecutive days) and oral cyclosporine $(2 \mathrm{mg} /$ $\mathrm{kg}$ /day). His respiratory status improved after the initiation of pharmacotherapy, and his nail coloration normalized (Fig. 1B). The patient was discharged uneventfully on hospital day 58.

In the follow-up period after discharge, the patient showed no respiratory symptoms; however, centrilobular nodules with or without consolidation were evident on thoracic CT at 1 (Fig. 2B) and 3 years (Fig. 2C) after discharge. The patient's sputum culture was found to be posi- tive for Streptococcus pneumoniae, and he was successfully treated with oral tosufloxacin $(450 \mathrm{mg} /$ day $)$. Interestingly, his nail color again became yellow at 4 years after discharge (Fig. 1C). At this time, although no lymphedema was evident in the patient's extremities, he was finally diagnosed with YNS based on the reappearance of yellow nails and his recurrent lower respiratory tract infections.

Six months after the diagnosis of YNS (4.5 years after his initial discharge), the patient was re-admitted to our hospital with dyspnea and a productive cough. On hospital day 2 , he 


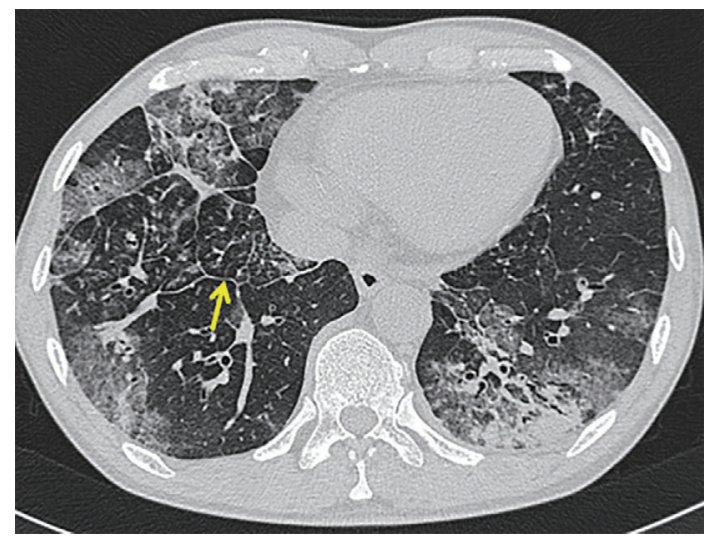

Figure 3. Chest computed tomography at 4.5 years after discharge shows consolidation with air bronchogram or groundglass opacities, centrilobular nodules, and accentuated interlobular septal thickening (arrow).

showed mild pyrexia $\left(37.1^{\circ} \mathrm{C}\right)$ with coarse crackles in the bilateral lower lung fields. No physical signs of heart failure or lymphedema in the extremities were noted. A laboratory investigation of the patient's serum revealed markedly elevated levels of C-reactive protein $(32 \mathrm{mg} / \mathrm{dL})$, but no apparent elevation of brain natriuretic protein $(32.9 \mathrm{pg} / \mathrm{mL})$. Thoracic CT showed the progression of lung lesions with various radiographic findings such as bilateral consolidation, scattered centrilobular nodules, traction bronchiectasis, and interlobular septal thickening, indicating lymphedema (Fig. 3). His sputum culture was positive for Haemophilus influenzae. He was thus diagnosed with recurrent bronchopneumonia and COP or organizing pneumonia (OP) accompanied by YNS. After starting treatment with intravenous sulbactam/ampicillin (12 g/day) with oral prednisolone (1 $\mathrm{mg} / \mathrm{kg} / \mathrm{day}$ ), the patient's lung lesions completely resolved, and the yellowish nail discoloration normalized.

\section{Discussion}

YNS was first described by Samman and White in 1964 (1). It is characterized by the triad of thickened yellow nails, lymphedema, and respiratory manifestations. Even though half a century has passed since it was first reported, the etiologies and underlying mechanisms of YNS remain unclear.

This case re-confirmed that YNS is a clinical condition that can be reversed with lung treatment using steroids over a long clinical course, as previously described (2). Another report showed that clarithromycin treatment for lung diseases ended in the complete resolution of yellow nails (3). Similarly, in the present case, oral prednisolone therapy for the patient's lung involvement was considered to be responsible for the normalization of his nail color; however, the precise extent and impact of prednisolone therapy on the progression of YNS are unknown, particularly in the context of lung involvement.

What is interesting to note in the present cases is that the yellow nails recurred 3 years after the first remission and improved again with the treatment. According to an analysis of 41 cases by Maldonado et al., yellow nails improved or resolved in 14 of the 25 cases (56\%) for whom follow-up data were available $(4,5)$. However, they did not mention any cases showing the reappearance of yellow nails. In this regard, the present case suggests that the yellow nails status can fluctuate over the long term.

Thus far, there is only one report of YNS in a Japanese patient. The case report indicates that the patient's lung involvement, in the form of OP, was successfully treated with steroids (6). Although we did not perform a pathological analysis at the patient's second admission to our hospital, our case suggests that an OP pattern on thoracic CT might represent a prominent radiological feature of YNS even in the early phase of the disease.

Doi et al. reported that only $63 \%$ of YNS patients show the complete triads of clinical manifestations (7). Indeed, the present case showed only two of the major clinical manifestations (recurrent lower tract infections and yellow nails) with no lymphedema recognized on the extremities. However, thoracic CT clearly demonstrated interlobular septal thickening (Fig. 3, arrow) which would have corresponded to lymphedema or impaired lymphatic drainage in the lung. The differential diagnoses for interlobular septal thickening on thoracic CT include pulmonary edema, lymphangitic carcinoma, and lymphoproliferative disorders (8). In the present case, lymphangitic carcinoma and lymphoproliferative disorders were excluded based on the fact that complete resolution of lung involvement was observed after treatment.

The present case re-confirmed that YNS is a reversible condition, and demonstrated that $\mathrm{OP}$ might represent a prominent feature of the lung involvement, even in the early phase of YNS. The accentuated interlobular septal thickening that was observed in the later phase would correspond to the phase of exaggerated lymphedema. Although the reason why the interlobular septal thickening only emerged in the later phase of the present case is unclear, this might represent a radiological clue for the diagnosis of YNS in the long-term observational period.

The authors are of the opinion that YNS could be considered as a differential diagnosis in cases showing interlobular septal thickening on thoracic CT, even in the presence of infectious manifestations.

The authors state that they have no Conflict of Interest (COI).

\section{References}

1. Samman PD, White WF. The "yellow nail" syndrome. Br J Dermatol 76: 153-157, 1964.

2. Scott J. Cardiac infarction and yellow nail syndrome. Proc R Soc Med 67: 323, 1974.

3. Suzuki M, Yoshizawa A, Sugiyama H, et al. A case of yellow nail syndrome with dramatically improved nail discoloration by oral clarithromycin. Case Rep Dermatol 3: 251-258, 2011.

4. Maldonado F, Tazelaar HD, Wang CW, Ryu JH. Yellow nail syn- 
drome: analysis of 41 consecutive patients. Chest 134: $375-381$, 2008.

5. Maldonado F, Ryu JH. Yellow nail syndrome. Curr Opin Pulm Med 15: 371-375, 2009.

6. Tanaka T, Yoshinaga T, Ushijima S, et al. A case of yellow nail syndrome accompanied by organizing pneumonia: a case successfully treated with steroid therapy. Nippon Kyobu Rinsho (Jpn J Chest Dis) 11: 961-968, 2007 (in Japanese, Abstract in English).
7. Doi R, Okamoto H, Horio T. Yellow nail syndrome with Major Triad and sinusitis treated with clarithromycin. Jpn J Dermatol 112: 261-265, 2002.

8. Webb WR, Higgins CB. Thoracic Imaging: Pulmonary and Cardiovascular Radiology. Plain Film and HRCT Assessment of Diffuse Infiltrative Lung Disease. Lippincott Williams \& Wilkins, Philadelphia, 2010: 330-331.

(C) 2016 The Japanese Society of Internal Medicine http://www.naika.or.jp/imonline/index.html 